\title{
Task Context Overrules Object- and Category-Related Representational Content in the Human Parietal Cortex
}

\author{
Stefania Bracci, Nicky Daniels and Hans Op de Beeck \\ Laboratory of Biological Psychology, KU Leuven 3000, Leuven, Belgium \\ Address correspondence to Stefania Bracci, Laboratorium voor Biologische Psychologie, Tiensestraat 102-bus 3714, 3000 Leuven, Belgium. \\ Email: stefania.bracci@kuleuven.be
}

\begin{abstract}
The dorsal, parietal visual stream is activated when seeing objects, but the exact nature of parietal object representations is still under discussion. Here we test 2 specific hypotheses. First, parietal cortex is biased to host some representations more than others, with a different bias compared with ventral areas. A prime example would be object action representations. Second, parietal cortex forms a general multiple-demand network with frontal areas, showing similar task effects and representational content compared with frontal areas. To differentiate between these hypotheses, we implemented a human neuroimaging study with a stimulus set that dissociates associated object action from object category while manipulating task context to be either action- or category-related. Representations in parietal as well as prefrontal areas represented task-relevant object properties (action representations in the action task), with no sign of the irrelevant object property (category representations in the action task). In contrast, irrelevant object properties were represented in ventral areas. These findings emphasize that human parietal cortex does not preferentially represent particular object properties irrespective of task, but together with frontal areas is part of a multiple-demand and content-rich cortical network representing task-relevant object properties.
\end{abstract}

Key words: dorsal stream, fMRI, object representations, representational similarity analysis, ventral stream

\section{Introduction}

Viewing objects activates areas in parietal cortex (Chao and Martin 2000; Konen and Kastner, 2008b; Freedman and Assad 2016; Jeong and Xu 2016), yet it is unclear what the exact function is of these activations in relationship to co-activated areas in ventral visual areas and frontal cortex (Chao and Martin 2000; Freedman et al. 2001, 2003; Konen and Kastner 2008a, 2008b). It already stands beyond doubt that object representations in human parietal cortex can be very rich in content (Freud et al. 2016). Earlier findings already reported view-, position-, and sizeinvariant object representations in parietal areas (Sereno and
Maunsell 1998). More recent studies provided an important extension to this earlier work by demonstrating through representational similarity analyses that parietal areas represent not just simple object features (Konen and Kastner 2008a, 2008b) or category boundaries (Freedman and Assad 2006), but even highly abstract object information such as face identity (Jeong and Xu 2016). Some of these findings are very reminiscent of what researchers would expect to find in ventral visual cortex (e.g., for face identity; Kriegeskorte et al. 2007; Nestor et al. 2011; Anzellotti et al. 2014) or in prefrontal cortex (Freedman et al. 2001, 2003). In what respects can we differentiate the properties and the

(C) The Author 2017. Published by Oxford University Press.

This is an Open Access article distributed under the terms of the Creative Commons Attribution Non-Commercial License (http://creativecommons.org/ licenses/by-nc/4.0/), which permits non-commercial re-use, distribution, and reproduction in any medium, provided the original work is properly cited. For commercial re-use, please contact journals.permissions@oup.com 
functions of the representations in parietal cortex from representations in the ventral visual stream and in frontal cortex?

The classic story is that the visual system is composed of a ventral occipitotemporal stream for object vision (Mishkin and Ungerleider 1982) and a dorsal occipitoparietal stream involved in perception for action (Milner and Goodale 1995), with both streams feeding into frontal cortex (Felleman and Van Essen 1991). Along the years, researchers have derived more specific hypotheses that are generally in line with this classic story, but that tend to make different predictions. A first hypothesis is that representations in the 2 visual streams should have a different content because some object properties are more relevant for object "what" vision and other properties are more relevant for action "where" perception. With this hypothesis as a background, it is understandable why researchers early on searched for identity coding in ventral areas and for position coding in dorsal areas (Haxby et al. 1991; Freiwald and Tsao 2010); why more recently Freedman et al. focused upon inferior temporal cortex for the categorization of dogs/cats (Freedman et al. 2003) and upon parietal cortex for the categorization of motion (Freedman and Assad 2006); and why it makes sense that objects tend to cluster along the animate/inanimate distinction in ventral cortex, and the action/nonaction distinction in parietal cortex (Bracci and Op de Beeck 2016). The finding of abstract face identity information by Jeong and $\mathrm{Xu}$ (2016) is a challenge to this hypothesis. However, the fact that it can represent such information under some task conditions does not exclude the possibility that parietal cortex would at least be biased toward representing some object properties more than other depending on their relevance for action.

A second hypothesis considers parietal areas to be part of a multiple-demand frontoparietal network (Duncan 2001; Duncan 2010; Woolgar et al. 2015), partially overlapping with well-known attention networks (Corbetta et al. 2000; Duncan 2001; Corbetta and Shulman 2002). These areas are recruited whenever subjects perform a relatively challenging task involving objects (or other stimuli). According to this hypothesis, we expect highly task-dependent representations in parietal cortex, high similarity between representations in parietal cortex and frontal cortex, and less similarity with representations in ventral cortex. Available evidence is in broad agreement to this view: dorsal stream areas represent learned category boundaries and dynamically switch representations depending on task relevance (Freedman et al. 2001; Freedman and Assad 2006; McKee et al. 2014; Erez and Duncan 2015), whereas information encoded within the ventral pathway largely reflects the stimulus' perceptual properties (Freedman and Assad 2006; Harel et al. 2014; McKee et al. 2014). In contrast to the first hypothesis, we do not expect much bias to intrinsically favor some object properties more than other. Nor do we expect any representation of task-irrelevant information, even not when it might often be useful for object-related action in other task contexts.

In the current literature, evidence for both views coexists but up to now no study has directly tested these 2 hypotheses against each other. This is what we do in the present human functional magnetic resonance imaging (fMRI) study. A stimulus set designed to dissociate object category from object action information was tested in 2 task contexts: 1) the category task targeted categorical relationship among objects shown to be represented in the ventral pathway, and 2) the action task targeted object hand-action/handmanipulation information typically associated with dorsal stream areas. Behavioral action and category similarity judgments were used to predict representational content across parietal, prefrontal, and occipitotemporal areas. The aforementioned hypotheses both predict task modulations in parietal cortex, but they differ in terms of the absoluteness of these task modulations and in terms of the relative similarity between occipitotemporal, parietal, and frontal areas. According to the first hypothesis, we expect parietal areas to preferentially encode object action information, and occipitotemporal areas to represent object category information, at least partially independent of task context. In addition, frontal areas might switch their representational space to a more ventral-stream-like or a more dorsal-stream-like representation depending on the task context. Conversely, the second hypothesis predicts dynamic changes in parietal and prefrontal representational content, with close to no representation of irrelevant object properties even when they are action-related. In addition, prefrontal areas would always be highly similar to parietal areas in terms of representational content. Our findings seem to align more with the second set of predictions.

\section{Materials and Methods}

\section{Participants}

Eighteen right-handed participants (18 females; mean age, 26 years) took part to the fMRI experiment. All participants gave informed consent following the procedures approved by the ethics committee of the KU Leuven. Two participants were excluded due to technical problems during data collection. Due to excessive head motion one run (out of 16) was excluded from analysis in 3 participants. All decisions about participant and run exclusion were taken prior or during preprocessing, thus before estimating the general linear models (GLMs).

\section{Experimental Design}

The whole study comprised a behavioral and an fMRI experiment. The study was divided in 2 separate sessions. A maximum period of 7 days was allowed between the first and the second session. Each session started with a behavioral experiment, followed by the fMRI experiment. The fMRI experimental design comprised a main experiment and 2 independent functional localizers.

\section{Behavioral Experiment}

Before each fMRI session, outside the scanner, participants performed similarity judgments (Kriegeskorte and Mur 2012) for the action- and category-related tasks. Each participant rated all 28 images used in the functional neuroimaging study. All images were simultaneously rated on a screen within a circle arena following the procedure of Kriegeskorte and Mur (2012), thus allowing measuring multiple similarity judgments in a single arrangement. For category-related judgments, participants were asked to "arrange the objects according to their semantic similarity." We know from our earlier work that the similarity according to such ratings is dominated by the category relationships among stimuli (Bracci and Op de Beeck 2016), which is why we refer to this task as the behavioral "category" task throughout the manuscript. For actionrelated judgments, participants were asked to "arrange the objects according to object hand action/manipulation similarities." For each task dimension (category or action), results are averaged across participants and sessions, and summarized in Fig. $1 \mathrm{C}$ by means of cluster analysis.

\section{fMRI Experiment}

Participants were presented with 28 grayscale images $(400 \times 400$ pixels) of everyday man-made objects (Fig. 1A) in an eventrelated design fMRI experiment divided in 2 separate sessions. 
A

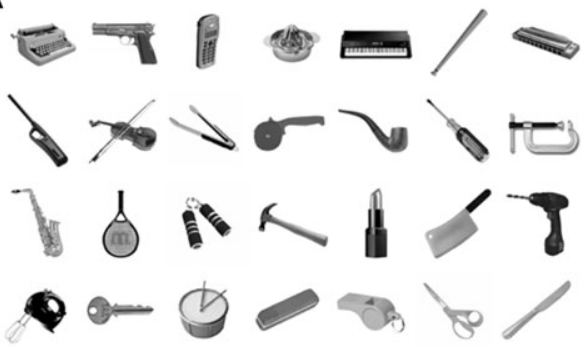

C

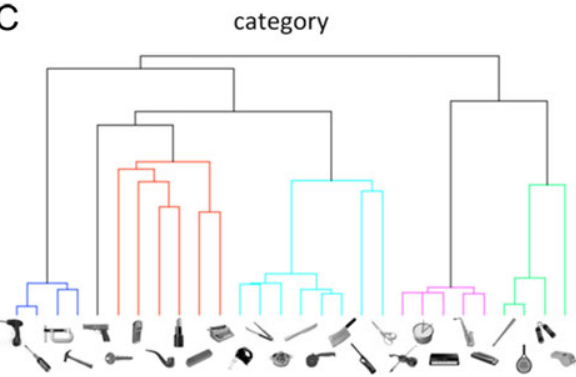

B

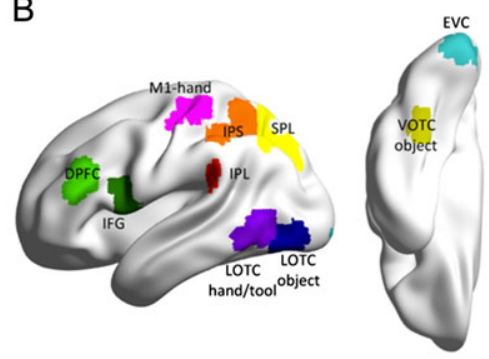

action

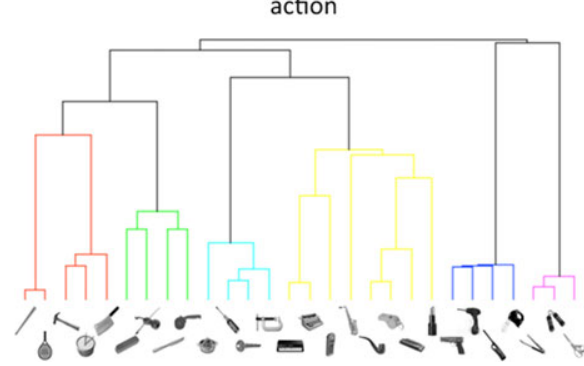

Figure 1. Experimental stimuli and behavioral results. (A) Of the note, 28 objects used for behavioral similarity judgments and for the event-related fMRI study. (B) Individual-participant ROIs are shown for the left hemisphere of one representative participant of the inflated human brain template with the BrainNet viewer (Xia et al. 2013). IPS, intraparietal sulcus; IPL, inferior parietal lobe; SPL, superior parietal lobe; DPFC, dorsal prefrontal cortex; IFG, inferior frontal gyrus; LOTC, lateral occipitotemporal cortex; VOTC, ventral occipitotemporal cortex; M1, primary motor cortex. (C) Based on behavioral similarity judgment (distance between objects arranged on a screen) hierarchical cluster analysis (nearest distance) was performed, averaged across participants and visualized as dendrograms.

Each session included 8 experimental runs (16 in total), each lasting 7 min and $52 \mathrm{~s}$. Within each run 4 fully randomized sequences of 28 image trials and 9 fixation trials were presented. Each trial was presented for $1500 \mathrm{~ms}$, followed by a fixation screen for $1500 \mathrm{~ms}$. Each run started and ended with $14 \mathrm{~s}$ of fixation. Counterbalanced across runs, participants performed 2 different tasks while maintaining fixation. In half of the runs participants judged object hand action/manipulation similarities, whereas in the remaining half they judged object semantic similarities through a 1-back similarity rating. More specifically, after each trial (during fixation) participants had to judge similarities between the current image and the one presented in the previous trial by pressing a response button (adopting a 4 responses scale ranging from "very similar" to "very different") using their right and left, middle and index finger. The fingers associated with each response were counterbalanced across runs.

It is worth noting that the representation of stimulus $\mathrm{N}$ during initial perception cannot be dissociated from the shortterm memory representation of the same stimulus $N(1.5 \mathrm{~s}$ later) during the ISI. Thus, the term "representational content" refers to the content of representations integrated across all processes that involve a representation of the stimulus that is independent of the preceding stimulus.

\section{Category Localizer}

Six categories of objects were included in a block-design fMRI localizer: animates (human and animal bodies), hands, tools, objects, places, and scrambled images. Each condition consisted of 18 grayscale images $(400 \times 400$ pixels) on a white background. In total, 4 visual localizer runs ( 2 runs for each session) were included in the study, each lasting $5 \mathrm{~min}$ and $36 \mathrm{~s}$. Within each run a fully randomized sequence of 6 category blocks (each repeated 5 times) interleaved with a fixation block lasting $16 \mathrm{~s}$ was presented. At the beginning and at the end of each run an additional fixation block was presented for $16 \mathrm{~s}$. Within each category block, a fully randomized sequence of 10 (out of 18) images was presented successively at the center of the screen. Each image was presented for $400 \mathrm{~ms}$ with a blank interstimulus interval (ISI) of $400 \mathrm{~ms}$ (block duration: $8 \mathrm{~s}$ ). Participants performed a 1-back repetition detection task by pressing a button with their right index finger any time the same picture was presented 2 times in succession. In each block, 1 or 2 repetitions were presented.

\section{Motor Localizer}

To localize the hand motor region in primary motor cortex we included a block-design fMRI motor localizer comprising 2 conditions: hand movements and foot movements. Each condition consisted of 2 different movements (circular, horizontal) in 2 directions (left, right). Within a single run, lasting $5 \mathrm{~min}$ and $50 \mathrm{~s}$, a randomized sequence of 2 condition blocks (each repeated 6 times) interleaved with a fixation block lasting $18 \mathrm{~s}$ was presented. At the beginning and at the end of the run an additional fixation block was presented for $14 \mathrm{~s}$. Each category block, started with $2 \mathrm{~s}$ cue indicating the effector to be used to perform movements (hand or foot). Subsequently, a sequence of 8 trials showing a white arrow on a black background indicated the direction and type of movement to be performed. Cues were presented at the center of the screen for $1 \mathrm{~s}$ followed by a fixation cross for $1 \mathrm{~s}$. Participants were asked to perform the movement indicated by the visual cue while fixating the central cross.

\section{Imaging Parameters and Apparatus}

Data collection was performed on a $3 \mathrm{~T}$ Philips scanner with a 32-channel coil at the Department of Radiology of the University Hospitals Leuven. MRI volumes were collected using echo planar T2*-weighted scans. Acquisition parameters were as follows: repetition time of $2 \mathrm{~s}$, echo time of $30 \mathrm{~ms}$, flip angle of $90^{\circ}$, field of view of $216 \mathrm{~mm}$, and matrix size of $72 \times 72$. Each volume comprised 37 axial slices (covering the whole brain) 
with $3 \mathrm{~mm}$ thickness and no gap. The T1-weighted anatomical images were acquired with an MP-RAGE sequence, with $1 \times 1 \times$ $1 \mathrm{~mm}$ resolution.

The stimuli presentation was controlled by a PC running the Psychophysics Toolbox package (Brainard 1997) in MATLAB. Pictures were projected onto a screen and were viewed through a mirror mounted on the head coil.

\section{fMRI Preprocessing}

Imaging data were preprocessed and analyzed with the Statistical Parametrical Mapping software package (SPM 8, Welcome Department of Cognitive Neurology) and MATLAB. Before statistical analysis, functional images underwent the following preprocessing steps: slice timing correction, spatial realignment (to the first image) to adjust for individual head motion, co-registration of functional and anatomical images, segmentation and spatial normalization to an MNI (Montreal Neurological Institute) template. Functional images were resampled to a voxel size of $3 \times 3 \times 3 \mathrm{~mm}$ and spatially smoothed by convolution of a Gaussian kernel of $4 \mathrm{~mm}$ fullwidth at half-maximum (Op de Beeck 2010). We modeled the preprocessed signal for each voxel, for each participant and for each of the 28 images using a GLM. For each subject, we estimated 2 independent GLMs, one for each task. The GLMs included regressors for each condition of interest (28 conditions) and the 6 motion correction parameters ( $x, y, z$ for translation and for rotation). Each predictor's time course was modeled for $3 \mathrm{~s}$ (stimulus presentation + fixation) by a boxcar function convolved with the canonical hemodynamic response function in SPM.

\section{Regions of Interest}

Regions of interest (ROIs) were defined in each individual participant throughout, parietal, prefrontal, and occipitotemporal cortices by means of an independent category localizer and the anatomical WFU PickAtlas Toolbox (Wake Forrest University PickAtlas, http://fmri.wfubmc.edu/cms/software). In parietal cortex, SPL and IPL were defined selecting all visually active voxels (all categories $>$ baseline) restricted to anatomical masking by Brodmann area BA-5/7 and BA-40, respectively. A more conservative contrast of hand and tool versus objects served to define IPS (Bracci et al. 2016). In prefrontal cortex, DPFC, and IFG were defined selecting all visually active voxels (all categories $>$ baseline) within the anatomical masks BA46 and BA44-45, respectively. Object-selective voxels were defined contrasting objects versus scrambled images in lateral and ventral occipitotemporal cortex (LOTC-object and VOTC-objects, respectively). In addition a region that has been shown to respond to both hands and tools was defined in lateral occipitotemporal cortex (LOTC-hands/tools; Bracci et al. 2012) contrasting hands and tools versus objects. Finally, the hand motor region in primary motor cortex (M1-hand), restricted to the anatomical mask BA4, was defined with the contrast of hand movements versus foot movements. These ROIs cover a large range of ventral (occipitotemporal) and dorsal (frontoparietal) brain areas; their localization was decided based on relevant literature and prior to performing any statistical analysis on the experimental data. Figure $1 B$ shows all ROIs in one representative participant.

Following the same procedure used in Bracci and Op de Beeck (2016), ROIs included all spatially contiguous voxels that exceeded the statistical uncorrected threshold $P<0.001$. When less than 20 active voxels were found at this threshold, a more liberal threshold of $P<0.01$ was applied. Only ROIs with at least 20 active voxels were included in an individual subject. To ensure that all ROIs were anatomically independent from each other a hierarchical voxel inclusion criterion was applied which reflected the hierarchy in functional criteria. For example, if a subset of object-selective voxels were also selective for hands, object-selective voxels were defined after excluding handselective voxels (those voxels where the response to hands was significantly higher than objects).

As ROI exclusion criterion we compared within-condition correlations (diagonal cells) with the average of betweencondition correlations (off-diagonal cells) to test whether in each ROI the response pattern conveyed information about stimulus identity. For this analysis, the matrices of the 2 tasks/ GLMs were averaged. Pairwise t-tests across participants revealed significant reliability of response patterns for each ROI $(P<0.05)$. Based on this criterion, all pre-defined ROIs were included in all subsequent analyses.

\section{fMRI Statistical Analysis}

For each voxel within a given ROI, parameter estimates for each task and condition (relative to baseline) were extracted for each participant and each run and normalized by subtracting the mean response across all conditions. Typically, ROIs showed a similar mean response in the 2 task conditions: in all ROIs overall mean activity did not differ across tasks $(P>0.003$, corrected for multiple comparisons). We therefore applied multivariate analyses to investigate whether ROI's fine-grained pattern activity changes under different task conditions. For each task, the full data set was divided 100 times into 2 random subsets of runs (set-1 and set-2) and the voxel response patterns for each object pair were correlated across these independent data sets. Correlations were averaged across the 100 iterations, thus resulting in an asymmetric $28 \times 28$ correlation matrix for each task, participant and ROI. For each correlation matrix, cells above and below the diagonal were averaged and only the upper triangle of the resulting symmetric matrix was used in the subsequent analyses. Correlation matrices were converted into dissimilarities matrices (1 minus correlation) and used as neural input for the RSA analysis (Kriegeskorte et al. 2008). As before (Op de Beeck et al. 2008), behavioral dissimilarity matrices for each model were correlated with the neural dissimilarity matrix of each ROI. Resulting correlations were Fisher transformed $\left\{0.5^{*} \log [(1+\mathrm{r}) /(1-\mathrm{r})]\right\}$ and tested with ANOVAs and pairwise t-tests.

To take into account the noise in the data, for each ROI we computed an estimate of the reliability of the data, which provides an indication of the maximum correlations we can expect given the signal to noise ratio of the data (Op de Beeck et al. 2008). For each subject and each ROI, the $28 \times 28$ correlation matrix was correlated with the averaged correlation matrix of the remaining participants. Values were averaged across participants. The resulting correlation values capture noise inherent to a single subject as well as noise caused by intersubject variability. This measure of reliability gives an estimate of the correlations we can expect in each ROI when correlating behavioral dissimilarity (e.g., action model) and neural dissimilarity (e.g., activation pattern in each ROI). Note however, that a reliability measure that focuses on between subject correlations might-in certain cases-underestimate the highest correlation expected. We should also note that reliability values reported in Figs 2 and 5 represent the averaged value across participants. Thus, variability across participants that to a certain extent 


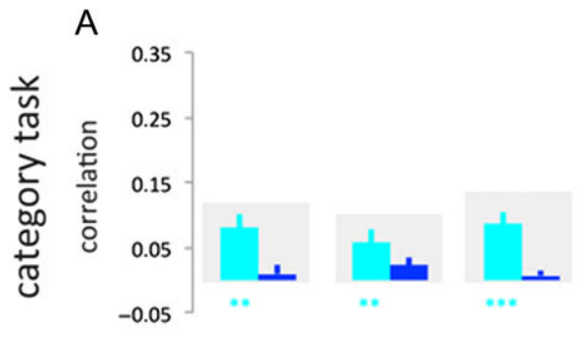

parietal areas

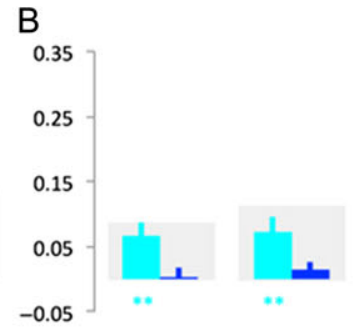

prefrontal areas

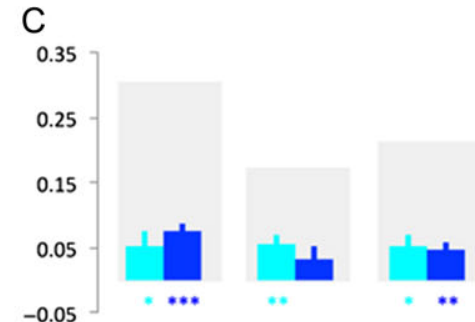

occipitotemporal areas

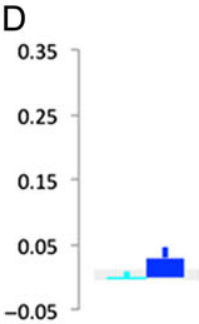

motor area

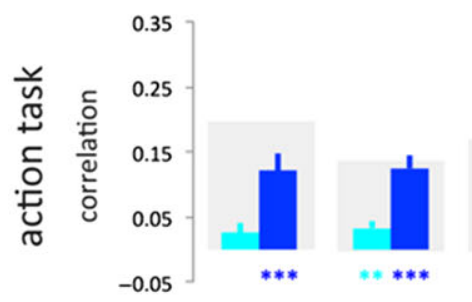

IPS

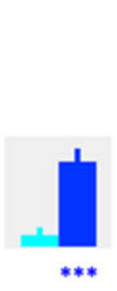

SPL
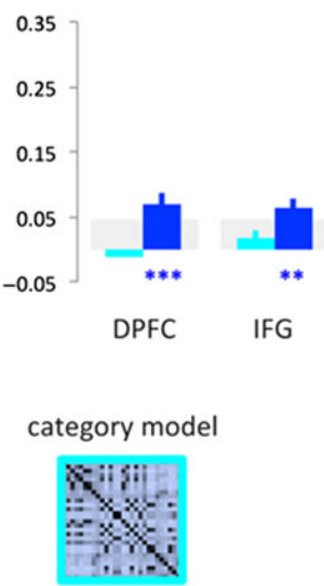
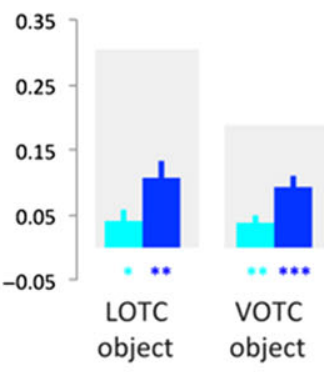

*.*.*

VOTC

object

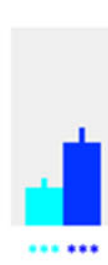

LOTC

hand/tool

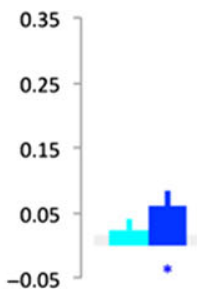

M1-hand

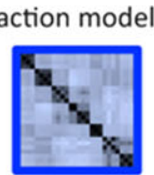

Figure 2. Representational similarity analysis (RSA) in parietal, prefrontal, and occipitotemporal areas. The ROI RSA results are shown for the category model (light blue) and the action model (dark blue) in the category task (top) and the action task (bottom). Findings are shown for (A) parietal, (B) prefrontal, (C) occipitotemporal areas, and (D) primary motor cortex. For each ROI, the gray-shaded background bar represents the reliability of the correlational patterns (see Materials and Methods), which provides an estimate of the highest correlation we can expect in a given ROI when correlating behavioral dissimilarity (e.g., category model) and neural dissimilarity (e.g., activation pattern). Error bars indicate SEM. IPS, intraparietal sulcus; IPL, inferior parietal lobe; SPL, superior parietal lobe; DPFC, dorsal prefrontal cortex; IFG, inferior frontal gyrus; LOTC, lateral occipitotemporal cortex; VOTC, ventral occipitotemporal cortex; M1, primary motor cortex. Asterisks indicate significance level $\left({ }^{* * *} \mathrm{P}<0.001,{ }^{* *} \mathrm{P}<0.01,{ }^{*} \mathrm{P}<0.05\right)$ for the category (light blue) and action (dark blue) model relative to baseline. The absence of asterisks implies that correlations do not differ from baseline.

might account for this discrepancy is not shown. Said otherwise, the reliability values are also an estimate with a confidence interval around it.

\section{Multidimensional Scaling and Hierarchical Cluster Analysis}

We employed multidimensional scaling (MDS) to visualize second level neural similarity structure across all ROIs for the action and semantic task. Metric MDS was performed using Matlab function "mdscale" normalized with the sum of squares of the dissimilarities. The hierarchical cluster, performed using the Matlab function "linkage" using the nearest distance default method, was used to visualize similarity structures derived from the behavioral tasks.

\section{Results}

We designed a stimulus set in which the semantic category of objects was dissociated from the action associated with each object (Fig. 1A). To measure the subjective perception of the fine-grained relationship among object category and action properties, participants provided similarity judgments on all images (Fig. 1A) by arranging objects on a screen (Kriegeskorte and Mur 2012). Resulting dissimilarities (1 minus correlation) describe the subjectively perceived representational structure of object category and action properties (see Materials and Methods). In this stimulus set, category- and action-related properties were unrelated at the behavioral level $(r=0.10$, $P>0.05)$. This is further illustrated by the clearly separated clusters, which emerged in both the category and the action task (Fig. 1C). In the category task, participants grouped together objects based on their fine-grained category-related relationships: utensils such as the food blender or the juicer were grouped together but separate from tools (e.g., screwdriver and drill). Conversely, in the action task, participants grouped together objects depending on their hand action/ manipulation similarities during object use: objects such as the screwdriver or the juicer, which require a similar rotating movement, were grouped together but separate from the food blender and the drill which both require to hold the object with a power grip while using the index finger to press a button.

Brain activity for all stimuli was measured in an eventrelated fMRI design while subjects performed a 1-back similarity judgment task with similar instructions as in the behavioral sessions. In half of the runs participants judged categoryrelated similarities, in the remaining half participants judged object action similarities (see Materials and Methods). Using data from independent localizers, individual ROIs were localized a priori by a combination of functionally and anatomically criteria (see Materials and Methods and Fig. 1B), including regions in parietal cortex (intraparietal sulcus, IPS; superior 
parietal lobe, SPL; and inferior parietal lobe, IPL), prefrontal cortex (dorsal prefrontal cortex, DPFC and inferior frontal gyrus, IFG) and VOTC and LOTC (object- and hand/tool-selective OTC).

To investigate changes in object representations as a consequence of task we used multivoxel pattern analysis (Norman et al. 2006) and RSA (Kriegeskorte and Kievit 2013). For each ROI and participant, we correlated the voxel response patterns for each object pair using independent data sets (see Materials and Methods) and resulting neural similarity matrices, one for each task context, were converted into a dissimilarity matrix (1 minus correlation). Then, we compared these matrices with the models (action and category) derived from behavioral judgments by means of RSA (see Materials and Methods). For each ROI, correlations between neural data and the 2 behavioral models were tested in a $2 \times 2$ ANOVA with fMRI Task (categorytask, action-task) and Model (category, action) as withinsubject factors.

\section{Object Representations Change Dynamically Based on Task in Parietal and Frontal Areas}

The object representations in parietal ROIs changed radically depending on the task performed (Fig. 2A). In all parietal ROIs, a highly significant Task $\times$ Model interaction $(F>14.56$, $P<0.002$, for all ROIs) revealed a striking double dissociation in representational content depending on task. That is, during the action task, object-related neural patterns were significantly correlated $(P<0.001$, for all ROIs) with the object hand-action/ hand-manipulation similarity structure as measured behaviorally (e.g., similar multivariate patterns for objects that are manipulated in a similar manner), with no evidence for object category information $(P>0.05$ for all ROIs; apart from IPL where the category model significantly differed from baseline $P=0.007)$. Conversely, when participants were engaged with the category task, the object fine-grained representational structure revealed significant similarities $(P<0.007$ for all ROIs $)$ with behaviorally perceived categorical divisions (e.g., similar multivariate patterns for objects within the same semantic category) with no evidence for object-hand action manipulation information $(P>0.10$, for all ROIs).

Similar results were observed in prefrontal areas (Fig. 2B). In both prefrontal ROIs there was a significant Task $\times$ Model interaction ( $F>12.88, P<0.003$, for both ROIs). During the action task, the neural activity patterns were significantly correlated with the action model ( $P<0.003$, for both ROIs), whereas correlations with the category model did not differ from baseline ( $P$ > 0.10; for both ROIs). The opposite pattern of results was observed for the category task. The category model could significantly explain activity patterns during the category task $(P<0.01$; for both ROIs) but the action model did not $(P>0.29$; for both ROIs).

Taken together, these results provide support for the second hypothesis suggesting that parietal areas, together with prefrontal areas, are part of a network recruited to represent any behaviorally relevant object property, including highly abstract object category information. Thus, in frontoparietal areas, representational content changes dynamically on a momentto-moment based depending on task context and represents task-relevant information (either object action or object category), with no trace of task-irrelevant information. These results are most striking for object hand-action information as this high-level property of objects is closely linked to the traditional view of the dorsal visual stream as a "perception for action" system. Thus, if any object property was to be represented in parietal cortex, even if not relevant for the current task, then it would be an object property such as hand action. Instead, the current results show that object action-related representations get lost when another property is task relevant, even if this other property is typically not associated with dorsal stream processing. These results support the view that, together with prefrontal areas, parietal areas are part of a multi-demand network (Duncan 2010) to represent whatever object property is relevant in a given behaviorally relevant situation.

To rule out the possibility that these results could be observed in any area, we tested the primary hand motor area (M1-hand) as control ROI (Fig. 2D). As expected, different results were observed in the primary motor area where we only observed a trend for a main effect of Model that did not reach significance $(t=2.91, P=0.11$ ). Irrespective of task, M1hand represented some action-related information (action task: $P=0.02$; semantic task: $P=0.07)$, but there was little evidence for category information $(P>0.19$, for both tasks). This task-independent representation of object action information makes it all the more striking that no object action information was retained in parietal areas when not relevant for the task.

\section{Task-Relevant and Task-Irrelevant Representations in Occipitotemporal Areas}

Different from frontoparietal representations, ventral ROIs showed representations of the task-irrelevant abstract object property (Fig. 2C). In high-level visual cortex, a significant Task $X$ Model interaction was observed in VOTC-obj $\left(F_{(1,15)}>10.58\right.$, $P=0.005)$. Thus, to a certain extent also occipitotemporal areas show some task-related effects (see also Harel et al. 2014). However, differently from frontoparietal areas, a significant above baseline correlations between neural data and both models $(P<0.03$, for all ROIs; a part from in VOTC-object where the action model did not differ from baseline $(P=0.15)$ in the category task) were observed irrespective of task. We can conclude that, in ventral areas, both object action and category information was present, at least to some degree, irrespective of task relevance.

The task-relevant object representations showed very similar correlations in occipitotemporal areas compared with frontoparietal areas (e.g., compare the light blue bars in the top row of Fig. 2). However, frontoparietal areas and occipitotemporal areas had a very different noise ceiling (the reliability estimates shown in the gray bars in Fig. 2) (Op de Beeck et al. 2008; Nili et al. 2014). Thus, task-relevant information was able to capture most of the signal variance in parietal and prefrontal areas, with empirical correlations close to the noise ceiling. Conversely, in occipitotemporal areas, correlations with either model were far below the noise ceiling. The discrepancy between empirical correlations and noise ceiling in occipitotemporal areas suggest that additional variables contribute to signal variance. Presumably, one of such a variable is object visual information. Additional tests of visual models (physical shape and perceived shape) confirmed that significant additional variance could be explained by object visual information in occipitotemporal areas (Figs 5 and 6).

To further investigate the differential task-related effects observed in dorsal and ventral areas, we directly compared correlations in dorsal (frontoparietal) and ventral (occipitotemporal) regions after averaging correlation values across dorsal ROIs (IPS-hand, IPL, SPL, DPFC, IFG) and across ventral ROIs (LOTC-object, VOTC-object, LOTC-hand/tool). Resulting 
correlations were tested in a $2 \times 2 \times 2$ ANOVA with Task (category-task, action-task), ROI (dorsal, ventral), and Model (category, action) as within-subject factors. A significant Task $\times$ ROI $\times$ Model interaction confirmed results showing differential task-related effects for dorsal and ventral object representational content $(F=11.01, P=0.01)$. Post hoc t-test confirmed that whereas in ventral occipitotemporal areas both taskrelevant and task-irrelevant information was significantly higher than baseline $(P<0.05$, for all tests), in dorsal frontoparietal areas only task-relevant information was significantly represented ( $P<0.02$ for both tasks) and task-irrelevant information did not differ from baseline ( $P>0.09$ for both tasks). Together, these results confirm previous analyses showing stronger taskrelated effects in frontoparietal relative to occipitotemporal areas.

To compare task-related changes in representational content across ROIs we performed second order correlations across ROI's correlation matrices averaged across subjects and visualized results by means of MDS (Fig. 3). Visual inspection of the
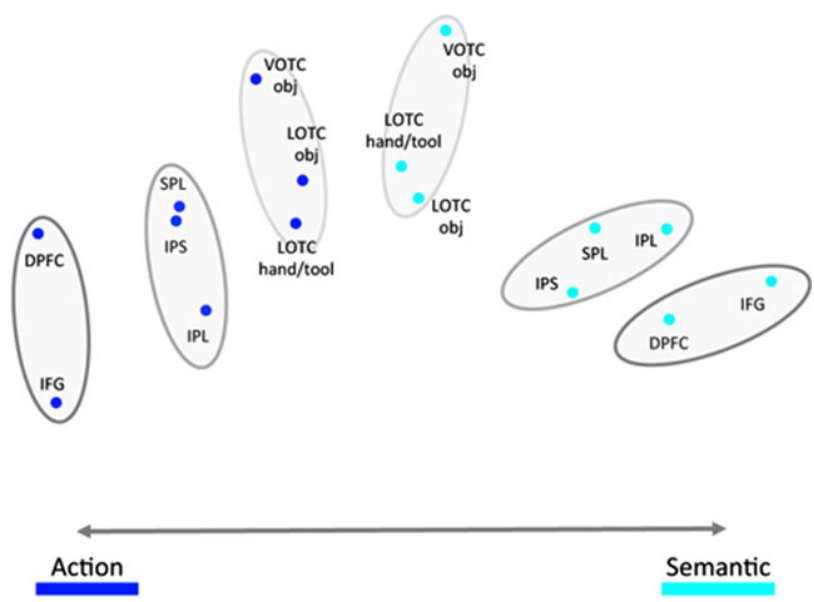

Figure 3. Task-related representational structure across ROIs. MDS, performed on second order correlations across ROI's correlation matrices averaged across subjects, shows ROIs pairwise distances in a 2D space. Differential task-related representational structure (action on the left side and category on the right side) emerges gradually from occipitotemporal areas to frontoparietal areas. For visualization purposes circles of different shades of gray highlight occipitotemporal (light gray), parietal (gray) and frontal (dark gray) areas.
MDS arrangement revealed similar within- and between-task distances across OTC areas, thus confirming that task has little influence on overall object representational content in occipitotemporal areas. Instead, increasing distance between the action-related (left side) and category-related (right side) representational space emerges throughout parietal and frontal areas, with an even stronger separation between tasks for frontal than for parietal areas. These results suggest that, although parietal and frontal areas belong to the same tasknetwork, there are differences in the overall object representational content (and potentially computational role) of these areas.

\section{Comparing Object Representational Space within and Across Tasks}

The object representational space in frontoparietal areas shifts strongly according to behavior relevance. Conversely, in occipitotemporal areas, object representational space seems to reflect individual objects irrespective of context. To further investigate task-related changes in regional representational content, we investigated how much the representational spaces change in a different task context by comparing individual object representations within-task versus betweentasks. That is, for each ROI and participant, we correlated the voxel response patterns for each object pair using independent data sets within-task (i.e., taking both data sets from one task) and between-tasks (i.e., taking one data set from the action task and one data set from the category task). For each ROI, we then computed within-condition correlations (diagonal cells) minus between-condition correlations (off-diagonal cells) for both within- and between-task matrices. Resulting correlations were tested using ANOVAs and independent $t$-tests (Fig. 4). Similar correlations across within- and between-task suggest that the multivariate pattern for individual objects is not influenced by context. Conversely, higher within-task correlations relative to between-task correlations suggest that representational space for individual objects changes dynamically depending on context.

In frotoparietal areas, a $2 \times 5$ ANOVA with Task Switch (within-task, between-task) and ROI (IPS, IPL, SPL, DPFC, IFG) as within-subject factors revealed a significant main effect of Task Switch $\left(F_{(1,12)}=18.77, P<0.001 ;\right.$ Fig. $\left.4 \mathrm{~A}\right)$. Further pairwise t-tests confirmed that in all dorsal ROIs within-task correlations were

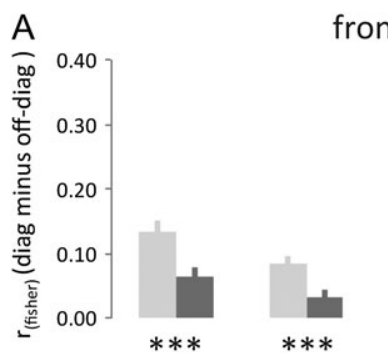

IPS frontoparietal

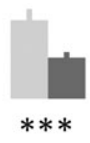

SPL

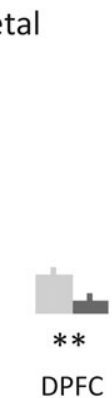

within task

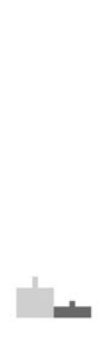

IFG

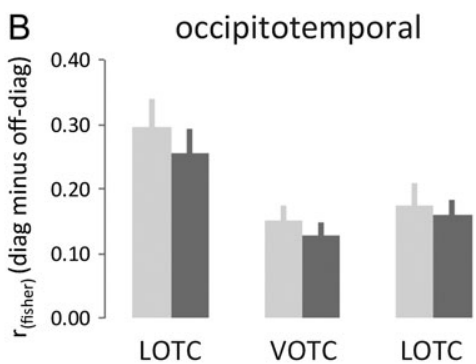

object object hand/tool

between task

Figure 4. Object representational space within and across tasks. Within-task correlations and between-task correlations are shown for (A) frontoparietal and (B) occipitotemporal ROIs. Error bars indicate SEM. IPS, intraparietal sulcus; IPL, inferior parietal lobe; SPL, superior parietal lobe; DPFC, dorsal prefrontal cortex; IFG, inferior frontal gyrus; LOTC, lateral occipitotemporal cortex; VOTC, ventral occipitotemporal cortex. Asterisks highlight significance level $\left({ }^{* * *} \mathrm{P}<0.001\right.$, $\left.{ }^{* *} \mathrm{P}<0.01,{ }^{*} \mathrm{P}<0.05\right)$ for within-task versus between-task comparisons. The absence of asterisks implies that correlations do not differ across tasks. 
significantly higher than between-task correlations $(P<0.005$, for all ROIs but IFG, $P=0.09$ ). Interestingly, the between-task correlations were still higher than zero in all ROIs $(P<0.05$, for all tests) but IFG $(P=0.09)$. Given that our earlier analyses (Fig. 2A,B) indicate that task-irrelevant object properties are not represented in these ROIs, these positive correlations might be related to object properties relevant in both task contexts. Finally, unsurprisingly, results revealed a significant main effect of ROI $\left(F_{(4,48)}=20.81, P<0.001\right)$ reflecting regional differences in the overall information conveyed about images.

In ventral areas, a $2 \times 3$ ANOVA with Task Switch (withintask, between-task) and ROI (LOTC-object, VOTC-object, LOTChand) as within-subject factors revealed neither a significant main effect of Task Switch $\left(F_{(2,22)}=3.46, P=0.09\right)$, nor a significant Task Switch $\times$ ROI interaction $(F<2$; Fig. $4 B)$. These findings confirm little or no influence of task in the fine-grained object representational content encoded in ventral stream regions. Similarly to dorsal ROIs, a significant main effect of ROI $\left(F_{(2,22)}=12.66, P<0.001\right)$ reflected regional differences in the overall information conveyed about images.

Although, this analysis does not inform on what aspects of object representations change across tasks, overall, these findings confirm the flexible role of frontoparietal representations in supporting task-relevant computations. Conversely, to sustain object recognition, occipitotemporal representations represent object visual and semantic properties mainly regardless of differential behavioral contexts.

\section{Object Shape Properties in Frontoparietal and Occipitotemporal Areas}

Our finding of strong task dependency in frontoparietal ROIs concerns relatively high-level and abstract object properties. Some more simple visual properties such as visual field location (retinotopic maps) and object size, and viewpoint invariance have been reported throughout the visual system, in both ventral and dorsal visual areas. These visual properties have a general relevance, independently of task context. We tested whether more basic visual dimensions are encoded in parietal, prefrontal, and occipitotemporal ROIs and to what extent these representations are also task dependent. In our stimulus set, the most obvious visual dimension relates to various aspects of object shape. Following the same procedure as in Bracci and Op de Beeck (2016), we computed the pixel-wise similarity between the images (physical shape model), and in addition obtained perceived shape judgments (perceived shape model) by asking participants to arrange objects according to object real-world shape similarities (Kriegeskorte and Mur 2012). Both visual models are shown in Fig. 5A by means of cluster analysis. Importantly, the correlations among all predictive models were small: category - perceived shape: $r=-0.03$; category - physical shape: $r=0.01$; action - perceived shape: 0.11 ; action - physical shape: $r=0.08$; perceived shape - physical shape: $r=-0.07$.

As before, for each ROI, correlations between neural data and the 2 shape models were tested in a $2 \times 2$ ANOVA with fMRI Task (category-task, action-task) and Model (physical shape, perceived shape) as within-subject factors. We found a main effect of Task in several frontoparietal ROIs $(P<0.02$; IPShand, SPL, and DPFC), but no Task $\times$ Model interaction $(F<2$, for all ROIs; Fig. 5B,C). In the action task, the neural similarity matrices in IPS-hand, IPL and SPL were correlated positively with the 2 shape models ( $P<0.01$; for both models). On the contrary, in the category-task neither shape model was significantly correlated with the neural data $(P>0.05$; for both models, in all ROIs but IPL for the perceived shape model, $P=0.03)$. These findings might reflect the partial correlation found between the action model and the 2 shape models $(r=0.08$ for the physical shape model, and $r=0.11$ for the perceived shape model), which was not observed for the category model ( $r=-0.03$ and 0.01, respectively).

Different results were observed in occipitotemporal ROIs (Fig. 5D) where the $2 \times 2$ ANOVA with Task (category-task, action-task) and Model (physical shape, perceived shape) as within-subject factors revealed a main effect of Model in all ROIs ( $F>18.96, P<0.001$, for all ROIs) but LOTC-object $(F<1)$. Confirming results from previous work (Op de Beeck et al. 2008; Bracci and Op de Beeck 2016), post hoc t-tests showed that early visual cortex (EVC: all categories > baseline; restricted to BA17) and regions in ventral visual cortex were very sensitive to visual shape properties, with a further progression from low-level shape to more high-level shape properties (Fig. 5D). Regardless of task, the representational content in occipitotemporal ROIs changed from reflecting object low-level physical form in early visual areas (physical shape model > perceived shape model: $P<0.001$, in EVC) to reflecting its subjective perceived shape in high-level visual areas (perceived shape model $>$ physical shape model: $P<0.05$, in VOTC-object and LOTC hand/tool). Together, these results confirm the role of ventral visual ROIs in representing a wide range of object properties: visual shape properties not directly related to our manipulation in task context, plus the aforementioned representation of more abstract category- and action-related object properties.

To rule out the possibility that differential task-related results, observed for the action and the semantic task (Fig. 2), could be explained by object shape properties, we tested all 4 models (physical shape, perceived shape, category, action) to the neural similarity matrix of each ROI using multiple regression. For each individual participant, we performed a multiple regression analysis with dissimilarity matrices for the 4 models as the dependent variable and an ROI's neural dissimilarity matrix as the independent variable. For each ROI, differences between the resulting regression coefficients were tested in a $2 \times 4$ ANOVA with fMRI Task (category-task, action-task) and Model (physical shape, perceived shape, semantic, action) as within-subject factors.

In all frontoparietal ROIs (Fig. 6A,B) we observed a significant Task $\times$ Model interaction $(F>4.99, P<0.005)$. Thus, confirming that object representations in the frontoparietal network change depending on the task at hand. The multiple regression analysis revealed that in the category task, only the category model was significantly positively related to the neural data $(P<0.01$, for all ROIs). Furthermore direct comparisons of the different models revealed that betas for the category model were significantly higher than betas for the action model $(P<$ 0.05 , for all ROIs). The opposite pattern of results was observed in the action task where betas were significantly higher for the action model relative to the category model $(P<0.05$, for all ROIs). However, in addition to the action model, here both shape models (physical shape and perceived shape) explained additional variance in parietal areas $(P<0.02$, for both models).

In occipitotemporal areas (Fig. $6 \mathrm{C}$ ), the multiple regression analysis revealed that both shape models were always positively related to the neural similarity $(P<0.02$, for all tests). In addition, in LOTC-hand/tool additional variance was explained by the 2 remaining models in both tasks $(P<0.05$, for all tests). Finally, in EVC and LOTC-object the action model could explain additional variance relative to object shape properties $(P<0.01$, for both tests). 
A

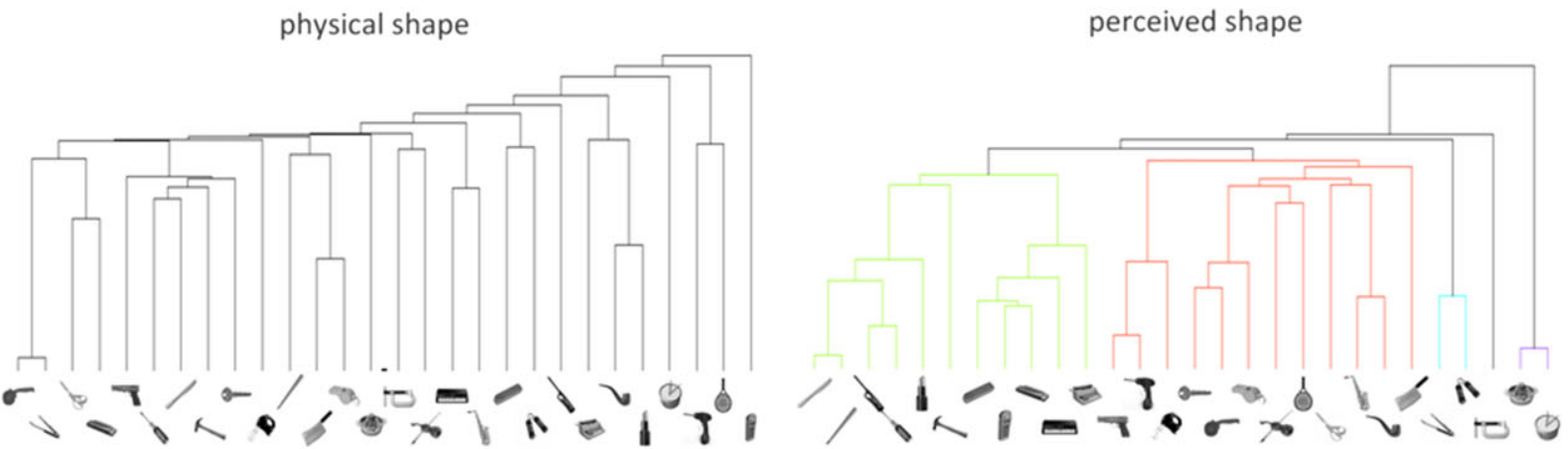

\section{B}

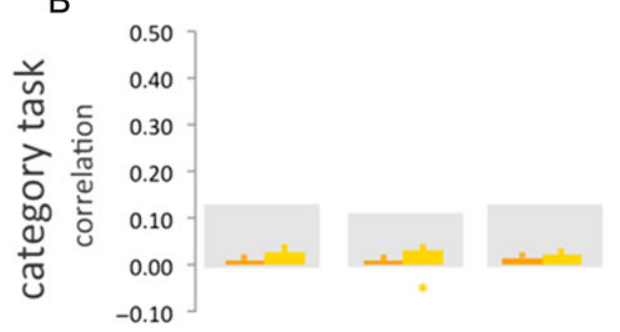

parietal areas
C

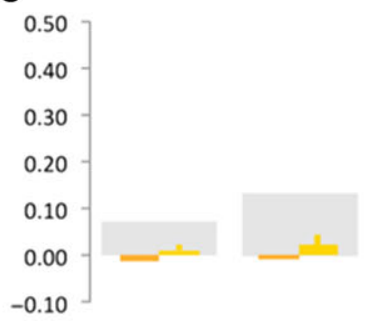

prefrontal areas

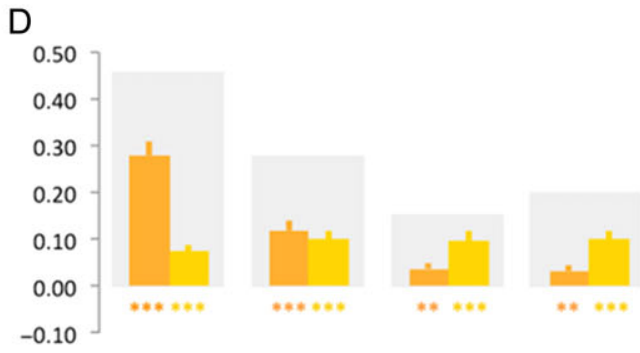

occipitotemporal areas

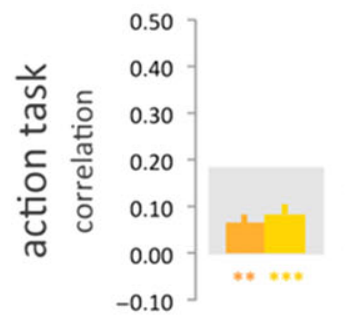

IPS

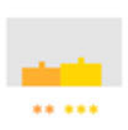

IPL

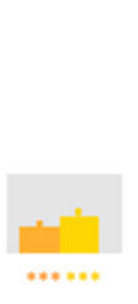

SPL

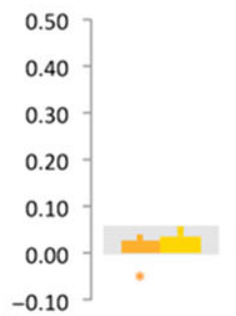

DPFC

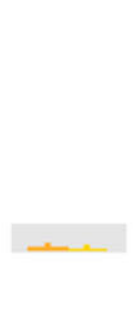

IFG

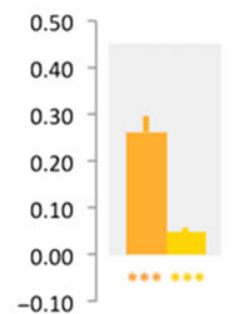

EVC

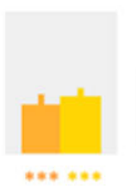

LOTC object

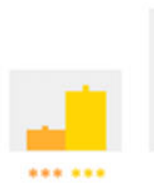

VOTC object

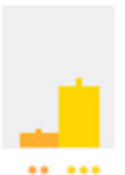

LOTC hand/tool physical shape model

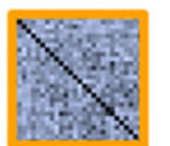

perceived shape model

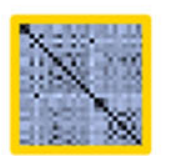

Figure 5. Object shape properties in parietal, frontal, and occipitotemporal areas. (A) Based on pixel-wise similarity (physical shape model) and behavioral similarity judgments (perceived shape model), the hierarchical cluster analysis (nearest distance) was performed (averaged across participants), and visualized as dendrograms. (B-D) The ROI RSA results for the physical shape model (orange) and the perceived shape model (yellow) in the category task (top) and the action task (bottom). Findings are shown for (A) parietal, (B) prefrontal, and (C) occipitotemporal areas. For each ROI, the gray-shaded background bar represents the reliability of the correlational patterns (see Materials and Methods), which provides an estimate of the highest correlation we can expect in each ROI when correlating behavioral dissimilarity (e.g., physical shape model) and neural dissimilarity (e.g., activation pattern). Error bars indicate SEM. IPS, intraparietal sulcus; IPL, inferior parietal lobe; SPL, superior parietal lobe; DPFC, dorsal prefrontal cortex; IFG, inferior frontal gyrus; EVC, early visual cortex; LOTC, lateral occipitotemporal cortex; VOTC, ventral occipitotemporal cortex. Asterisks indicate significance level $\left({ }^{* * *} \mathrm{P}<0.001,{ }^{* *} \mathrm{P}<0.01,{ }^{*} \mathrm{P}<0.05\right)$ for the physical shape model (orange) and the perceived shape model (yellow) relative to baseline. The absence of asterisks indicates that correlations do not differ from baseline.

\section{Discussion}

Object representations have been reported in both visual pathways; here we show differences in their purpose and information content. Whereas occipitotemporal areas encode object properties irrespective of task, and task has small effects on the overall object information content, frontoparietal areas represent task-relevant information only, showing dynamic changes in their information content, which reflected participants' behavior. Most surprisingly, even when it comes to object action, for which many studies have previously reported activations within the frontoparietal network (for review see, Lewis 2006), our results show no trace of actionrelated representations within dorsal areas unless this object knowledge is task-relevant. Finally, our analyses revealed both similarities and differences between prefrontal and parietal representations, thus suggesting that both regions represent taskrelevant information but their computations might differ. 


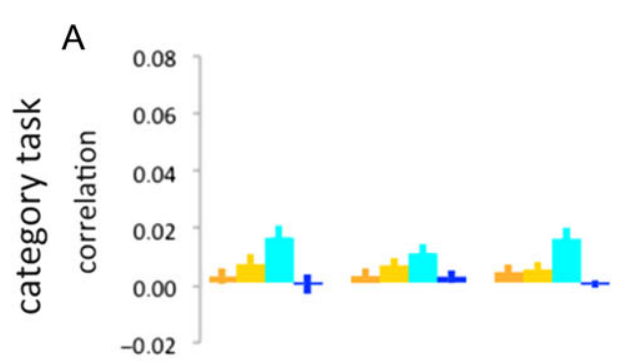

parietal areas

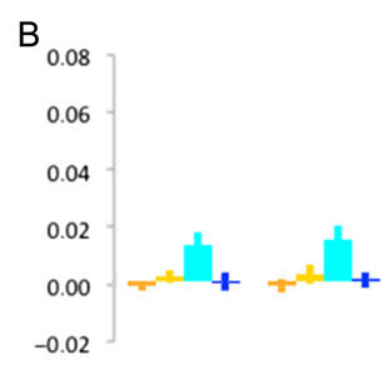

prefrontal areas

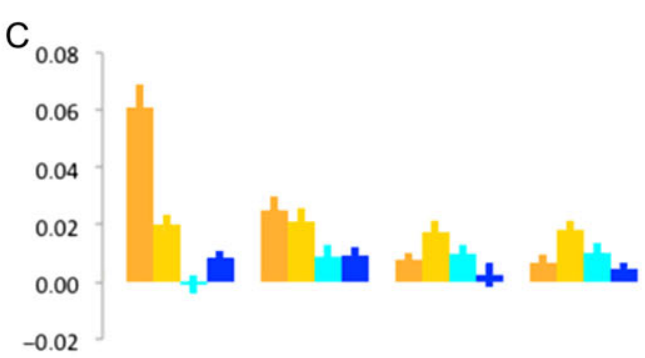

occipitotemporal areas

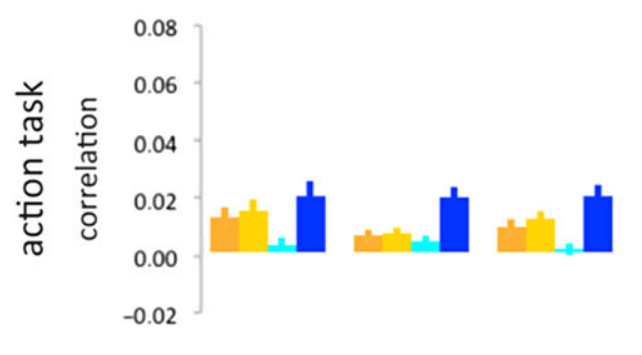

IPS

IPL $\quad$ SPL
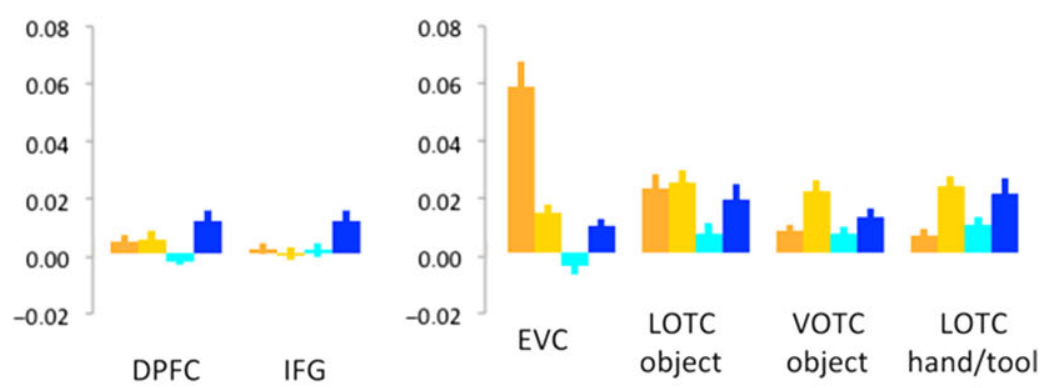

physical shape model

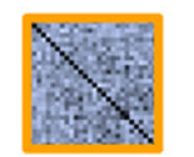

perceived shape model

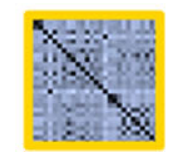

category model

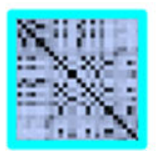

action model

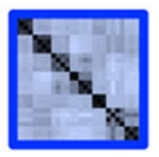

Figure 6. RSA in parietal, prefrontal, and occipitotemporal areas for all models. (A) For parietal, (B) prefrontal, and (C) occipitotemporal ROIs, bar graphs show parameter estimates of regression analyses relating model-based dissimilarity matrices (physical shape, perceived shape, category, action) to neural dissimilarity matrices for the category task (top) and the action task (bottom). Error bars indicate SEM. IPS, intraparietal sulcus; IPL, inferior parietal lobe; SPL, superior parietal lobe; DPFC, dorsal prefrontal cortex; IFG, inferior frontal gyrus; EVC, early visual cortex; LOTC, lateral occipitotemporal cortex; VOTC, ventral occipitotemporal cortex.

Our study demonstrates that parietal and prefrontal cortex can represent complex high-level semantic object properties, thus providing an important extension for findings reporting object category representations in frontoparietal cortex (Konen and Kastner 2008a, 2008b; McKee et al. 2014; Jeong and Xu 2016). For instance in Jeong and $X u$ (2016), the authors suggest that, similar to occipitotemporal areas, parietal areas can contain surprisingly invariant representations of highly abstract objects such as faces or cars. These studies did not rule out that such invariant representations would be highly or even entirely task dependent. In fact, such task dependence was shown earlier using a relatively small number of stimuli (Freedman et al. 2001; Erez and Duncan 2015) or simple stimulus dimensions (Crittenden and Duncan 2014) while manipulating task rules or task difficulty (Fedorenko et al. 2013; Woolgar et al. 2013). Another recent study (Nastase et al. 2016) showed task modulations in parietal cortex during the observation of actions, although in that case part of the effects might also be caused by attention to a relatively simple stimulus dimension, visual motion, which is almost by definition confounded with attending or not attending to observed actions.

In the current study, we extend these earlier findings to more abstract object representations such as category membership and action knowledge and show that frontoparietal representations reflect fine-grained object structural similarity as measured by complex behavior. By demonstrating such abstract representations in parietal cortex as well as their task dependence we provide 2 crucial new pieces of evidence that help to differentiate among different hypotheses about how to conceptualize the role of parietal cortex. First, parietal cortex can represent very complex object properties of a wide variety, including abstract semantic properties. Second, these abstract representations are highly and almost exclusively task relevant, even when involving an action-related object property that has been theorized to be linked with parietal cortex (Buxbaum et al. 2007). Together, these results provide important evidence for how parietal areas act as a hub of regions to sustain complex behavior, adapting their content flexibly moment by moment to meet changes in task requirements (Duncan 2001, 2010).

Earlier lesion and functional neuroimaging studies suggested the involvement of frontoparietal areas in sustaining tool-use and hand-object manipulation skills (Chao and Martin 2000; Buxbaum et al. 2007). In particularly, lesions involving frontoparietal areas do frequently lead to limb apraxia (Buxbaum et al. 2007; Ietswaart and Evans 2014) an acquired neuropsychological disorder that affects the ability to plan and execute manual actions (Liepmann 1900). Yet, the specific role of these regions in action recognition and execution remained unclear. Recent lesions studies converge suggesting that (tool) action representations might be stored in temporal areas (Kalenine et al. 2010; Buxbaum et al. 2014) and retrieved by frontoparietal areas when 
needed. In a recent voxel-based lesion study (Buxbaum et al. 2014), which included an extremely large number $(>70)$ of stroke patients, the authors revealed that differently from what earlier theories assumed, impaired access to tool-use knowledge was critically associated with lesions in the posterior middle temporal area rather than parietal areas. These results are also in agreement with earlier evidence showing that focal lesions within the left posterior middle temporal gyrus drastically impaired semantic knowledge of actions such as judging hand action properties (e.g., tiredness) or matching photographs depicting actions with corresponding verbs (Tranel et al. 2003; Kemmerer et al. 2012). To summarize, our results targeting object action semantics in task-relevant and taskirrelevant contexts, provide complementary evidence to these recent lesions studies pointing to the role of frontoparietal areas in action retrieval and planning, rather than being directly involved in the storage of hand action representations (but see also Klaes et al. 2015).

Differently from dorsal stream areas, task had more minor effects on ventral stream representations. Across all ventral stream ROIs, the overall representational space did not change across tasks (Fig. 4B). These results might in part reflect the dominant effect of object visual properties represented in ventral areas (Fig. 5D). This observation is in line with recent studies (Kuhl et al. 2013; McKee et al. 2014) reporting significantly stronger task-dependent modulations in prefrontal areas relative to ventral occipital areas, although weak effects of task were also observed in the ventral pathway. Some minor task effects (maybe related to feedback activity; Harel et al. 2014) might be missed in ventral stream areas in our study (there was a small not significant trend in this direction; Fig. 4B). Nevertheless, together results confirm a marked difference in terms of task-related effects between dorsal and ventral areas: the former being primarily modulated by task relevance, the latter being primarily involved in perceptual object processing.

To sum, by manipulating task context while dissociating 2 theoretically highly relevant object properties, object category and object-related actions, we could test 2 hypotheses concerning the representational content of high-level object properties in parietal cortex. Results showed that frontoparietal areas represent task-relevant information almost exclusively, thus altering information content as consequence of environmental needs. On the other hand, object representations in occipitotemporal areas are less influenced by task context. Differential ventral and dorsal representations are likely necessary to sustain different brain processing: whereas occipitotemporal areas play a vital role in object recognition with minor influence of behavioral relevance, frontoparietal areas flexibly adapt their representational content to achieve goal directed behavior in a continuous changing environment. These frontoparietal representations are content-rich, but lack across-task stability.

\section{Funding}

This work was supported by the European Research Council (ERC-2011-Stg-284101), a federal research action (IUAP-P7/11) and Hercules grant ZW11_10.

\section{Notes}

We thank M. Peelen and C. Baker for useful discussions. Conflict of Interest: None declared.

\section{References}

Anzellotti S, Fairhall SL, Caramazza A. 2014. Decoding representations of face identity that are tolerant to rotation. Cereb Cortex. 24:1988-1995.

Bracci S, Cavina-Pratesi C, Connolly JD, Ietswaart M. 2016. Representational content of occipitotemporal and parietal tool areas. Neuropsychologia. 84:81-88.

Bracci S, Cavina-Pratesi C, Ietswaart M, Caramazza A, Peelen MV. 2012. Closely overlapping responses to tools and hands in left lateral occipitotemporal cortex. J Neurophysiol. 107: 1443-1456.

Bracci S, Op de Beeck H. 2016. Dissociations and associations between shape and category representations in the two visual pathways. J Neurosci. 36:432-444.

Brainard DH. 1997. The psychophysics toolbox. Spat Vis. 10: 433-436.

Buxbaum LJ, Kyle K, Grossman M, Coslett HB. 2007. Left inferior parietal representations for skilled hand-object interactions: evidence from stroke and corticobasal degeneration. Cortex. 43:411-423.

Buxbaum LJ, Shapiro AD, Coslett HB. 2014. Critical brain regions for tool-related and imitative actions: a componential analysis. Brain. 137:1971-1985.

Chao LL, Martin A. 2000. Representation of manipulable man-made objects in the dorsal stream. Neuroimage. 12: 478-484.

Corbetta M, Kincade JM, Ollinger JM, McAvoy MP, Shulman GL. 2000. Voluntary orienting is dissociated from target detection in human posterior parietal cortex. Nat Neurosci. 3: 292-297.

Corbetta M, Shulman GL. 2002. Control of goal-directed and stimulus-driven attention in the brain. Nat Rev Neurosci. 3: 201-215.

Crittenden BM, Duncan J. 2014. Task difficulty manipulation reveals multiple demand activity but no frontal lobe hierarchy. Cereb Cortex. 24:532-540.

Duncan J. 2001. An adaptive coding model of neural function in prefrontal cortex. Nat Rev Neurosci. 2:820-829.

Duncan J. 2010. The multiple-demand (MD) system of the primate brain: mental programs for intelligent behaviour. Trends Cogn Sci. 14:172-179.

Erez Y, Duncan J. 2015. Discrimination of visual categories based on behavioral relevance in widespread regions of frontoparietal cortex. J Neurosci. 35:12383-12393.

Fedorenko E, Duncan J, Kanwisher N. 2013. Broad domain generality in focal regions of frontal and parietal cortex. Proc Natl Acad Sci U S A. 110:16616-16621.

Felleman DJ, Van Essen DC. 1991. Distributed hierarchical processing in the primate cerebral cortex. Cereb Cortex. 1:1-47.

Freedman DJ, Assad JA. 2006. Experience-dependent representation of visual categories in parietal cortex. Nature. 443:85-88.

Freedman DJ, Assad JA. 2016. Neuronal mechanisms of visual categorization: an abstract view on decision making. Annu Rev Neurosci. 39:129-147.

Freedman DJ, Riesenhuber M, Poggio T, Miller EK. 2001. Categorical representation of visual stimuli in the primate prefrontal cortex. Science. 291:312-316.

Freedman DJ, Riesenhuber M, Poggio T, Miller EK. 2003. A comparison of primate prefrontal and inferior temporal cortices during visual categorization. J Neurosci. 23:5235-5246.

Freiwald WA, Tsao DY. 2010. Functional compartmentalization and viewpoint generalization within the macaque faceprocessing system. Science. 330:845-851. 
Freud E, Plaut DC, Behrmann M. 2016. "What" is happening in the dorsal visual pathway. Trends Cogn Sci. 20:10.

Harel A, Kravitz DJ, Baker CI. 2014. Task context impacts visual object processing differentially across the cortex. Proc Natl Acad Sci U S A. 111:E962-E971.

Haxby JV, Grady CL, Horwitz B, Ungerleider LG, Mishkin M, Carson RE, Herscovitch P, Schapiro MB, Rapoport SI. 1991. Dissociation of object and spatial visual processing pathways in human extrastriate cortex. Proc Natl Acad Sci U S A. 88:1621-1625.

Ietswaart M, Evans C. 2014. In search for the core of apraxia. Cortex. 57:283-285. discussion 306-288.

Jeong SK, Xu Y. 2016. Behaviorally relevant abstract object identity representation in the human parietal cortex. J Neurosci. 36:1607-1619.

Kalenine S, Buxbaum LJ, Coslett HB. 2010. Critical brain regions for action recognition: lesion symptom mapping in left hemisphere stroke. Brain. 133:3269-3280.

Kemmerer D, Rudrauf D, Manzel K, Tranel D. 2012. Behavioral patterns and lesion sites associated with impaired processing of lexical and conceptual knowledge of actions. Cortex. 48:826-848.

Klaes C, Kellis S, Aflalo T, Lee B, Pejsa K, Shanfield K, HayesJackson S, Aisen M, Heck C, Liu C, et al. 2015. Hand shape representations in the human posterior parietal cortex. J Neurosci. 35:15466-15476.

Konen CS, Kastner S. 2008a. Representation of eye movements and stimulus motion in topographically organized areas of human posterior parietal cortex. J Neurosci. 28:8361-8375.

Konen CS, Kastner S. 2008b. Two hierarchically organized neural systems for object information in human visual cortex. Nat Neurosci. 11:224-231.

Kriegeskorte N, Formisano E, Sorger B, Goebel R. 2007. Individual faces elicit distinct response patterns in human anterior temporal cortex. Proc Natl Acad Sci U S A. 104: 20600-20605.

Kriegeskorte N, Kievit RA. 2013. Representational geometry: integrating cognition, computation, and the brain. Trends Cogn Sci. 17:401-412.

Kriegeskorte N, Mur M. 2012. Inverse MDS: inferring dissimilarity structure from multiple item arrangements. Front Psychol. 3:245.

Kriegeskorte N, Mur M, Bandettini P. 2008. Representational similarity analysis - connecting the branches of systems neuroscience. Front Syst Neurosci. 2:4.

Kuhl BA, Johnson MK, Chun MM. 2013. Dissociable neural mechanisms for goal-directed versus incidental memory reactivation. J Neurosci. 33:16099-16109.
Lewis JW. 2006. Cortical networks related to human use of tools. Neuroscientist. 12:211-231.

Liepmann H. 1900. Das krankheitsbild der apraxie. Monatsschr Psychiatr Neurol. 8:102-132.

McKee JL, Riesenhuber M, Miller EK, Freedman DJ. 2014. Task dependence of visual and category representations in prefrontal and inferior temporal cortices. J Neurosci. 34: 16065-16075.

Milner AD, Goodale MA. 1995. The visual brain in action. Oxford: Oxford University Press.

Mishkin M, Ungerleider LG. 1982. Contribution of striate inputs to the visuospatial functions of parieto-preoccipital cortex in monkeys. Behav Brain Res. 6:57-77.

Nastase SA, Connolly AC, Oosterhof NN, Halchenko YO, Guntupalli JS, Visconti di Oleggio Castello M, Gors J, Gobbini MI, Haxby JV. 2016. Attention selectively reshapes the geometry of distributed semantic representation. bioRxiv.

Nestor A, Plaut DC, Behrmann M. 2011. Unraveling the distributed neural code of facial identity through spatiotemporal pattern analysis. Proc Natl Acad Sci U S A. 108: 9998-10003.

Nili H, Wingfield C, Walther A, Su L, Marslen-Wilson W, Kriegeskorte N. 2014. A toolbox for representational similarity analysis. PLoS Comput Biol. 10:e1003553.

Norman KA, Polyn SM, Detre GJ, Haxby JV. 2006. Beyond mindreading: multi-voxel pattern analysis of fMRI data. Trends Cogn Sci. 10:424-430.

Op de Beeck HP. 2010. Against hyperacuity in brain reading: spatial smoothing does not hurt multivariate fMRI analyses? Neuroimage. 49:1943-1948.

Op de Beeck HP, Torfs K, Wagemans J. 2008. Perceived shape similarity among unfamiliar objects and the organization of the human object vision pathway. J Neurosci. 28: 10111-10123.

Sereno AB, Maunsell JH. 1998. Shape selectivity in primate lateral intraparietal cortex. Nature. 395:500-503.

Tranel D, Kemmerer D, Adolphs R, Damasio H, Damasio AR. 2003. Neural correlates of conceptual knowledge for actions. Cogn Neuropsychol. 20:409-432.

Woolgar A, Bor D, Duncan J. 2013. Global increase in taskrelated fronto-parietal activity after focal frontal lobe lesion. J Cogn Neurosci. 25:1542-1552.

Woolgar A, Williams MA, Rich AN. 2015. Attention enhances multi-voxel representation of novel objects in frontal, parietal and visual cortices. Neuroimage. 109:429-437.

Xia M, Wang J, He Y. 2013. BrainNet Viewer: a network visualization tool for human brain connectomics. PLoS One. 8: e68910. 